\title{
Ulcerated White Lesion of Tongue in a 7-Year-Old Child: A Diagnostic Dilemma
}

\author{
Royasa Shakya, ${ }^{1}$ Bandana Koirala,${ }^{2}$ Mamta Dali, ${ }^{3}$ Sneha Shrestha ${ }^{4}$ \\ ${ }^{1}$ Junior Resident, ${ }^{2}$ Professor, ${ }^{3}$ Associate Professor, ${ }^{4}$ Assistant Professon \\ ${ }^{1-4}$ Department of Pedodontics and Preventive Dentistry, College of Dental Surgery, \\ B.P. Koirala Institute of Health Sciences, Dharan, Sunsari, Nepal.
}

\begin{abstract}
Tongue is a complex organ associated with functions of taste, speech, mastication, deglutition, and development of jaws. It is considered the mirror of physical health as it can be the site of infections,immunological and drug-induced reactions,neoplasms and systemic diseases. This paper aims to present challenges in diagnosing a case of a 7-year-old male child who presented with ulceration and extensive white coating on the dorsum of tongue accompanied by fever,chills,tonsillitis,and submandibular lymphadenopathy. Despite symptomatic treatment done,the white coating with patches of depapillation lasted for a relatively long time. This may be the first reported case in the literature with such unusual tongue lesions in a child till date. Thus,pediatric dentists should have sound knowledge of the commonly encountered oral soft tissue lesions along with the rarer lesions and unusual presentation of common lesions for timely diagnosis,appropriate treatment,and referral if necessary.
\end{abstract}

Key words: Benign migratory glossitis; coated tongue; PFAPA syndrome; primary herpetic gingivostomatitis; tongue ulceration; tonsillitis.

\section{INTRODUCTION}

The prevalence of tongue lesions as reported by various epidemiological studies conducted in different parts of the world and approximately up to $18.5 \%$ has been found. ${ }^{1}$ Tongue ulceration can be seen as the manifestation of a spectrum of conditions. These ranges from infectious diseases such as herpesviruses, HIV, tuberculosis, candidiasis; dermatological diseases as lichen planus, pemphigus, pemphigoid; some gastrointestinal diseases; nutritional deficiencies; drug-induced reactions, and traumatic ulcers. ${ }^{2}$ White coated tongue is usually associated with local factors such as poor oral hygiene and the character of diet. It may also appear in systemic diseases with associated fever and dehydration. ${ }^{3}$

\section{CASE DESCRIPTION}

A 7-year-old male patient was referred to the Department of Pedodontics and Preventive Dentistry, BPKIHS,

Correspondence

Dr. Royasa Shakya

Department of Pedodontics and Preventive Dentistry,B.P.

Koirala Institute of Health Sciences,Dharan,Nepal

E-mail: royasashk@gmail.com

\section{Citation}

Shakya R, Koirala B, Dali M, Shrestha S. Ulcerated White Lesion of Tongue in a 7-Year-Old Child: A Diagnostic Dilemma. J Nepal Assoc Pediatr Dent. 2021;2(1):41-4.
Dharan with chief complaint of a whitish coating on the dorsal surface of tongue which was painful on eating and swallowing for five days. There was a history of fever in the last seven days and patient was taken to the local hospital where enlarged tonsils were seen on examination and diagnosis of acute tonsillitis with septicemia was made. The patient was admitted to the hospital and kept under antibiotic therapy. Two days after the onset of fever, ulcerative lesions started appearing on the tongue with a whitish coating which gradually increased in size. The patient experienced pain in swallowing with a burning sensation on eating. With no improvement in the oral lesions, the patient was referred to BPKIHS. The patient had no history of similar lesions in the past, but was hospitalized 2 years ago for fever and had a history of tonsillitis 4 years back.

On clinical examination, white coating that was not scrapable with gauge piece was present on the dorsal surface of the tongue along with multiple ulcers covered with yellowish pseudomembrane (Figure 1). Multiple coalesced ulcers of varying sizes were seen on the hard palate,as well with an erythematous border and yellowish base (Figure 2). The patient had poor oral hygiene with marginal and papillary gingivitis. Multi ple carious teeth 54, $51,61,74,84,85$ and root stumps of 64,75 were present. Bilateral submandibular lymph nodes were palpable, mobile,and non-tender. 


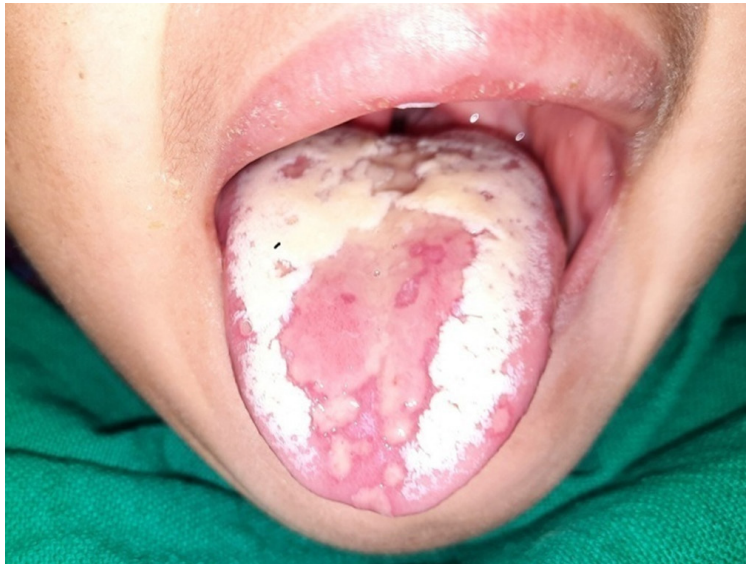

Figure 1. White non-scrapable coating with multiple ulcers on dorsal surface of tongue.

A routine blood investigation, peripheral smear and serological tests were done along with an overall examination of the child by a Pediatrician. On laboratory investigations, erythrocyte sedimentation rate (ESR) was elevated, C-reactive protein (CRP) was high and white blood cell (WBC) count was increased. Exfoliative cytology was performed, cytological smear prepared and Periodic-Acid-Schiff (PAS) staining was done which showed bacterial cocci with no presence of fungal hyphae. The serology test for Human Immunodeficiency Virus (HIV) was negative.

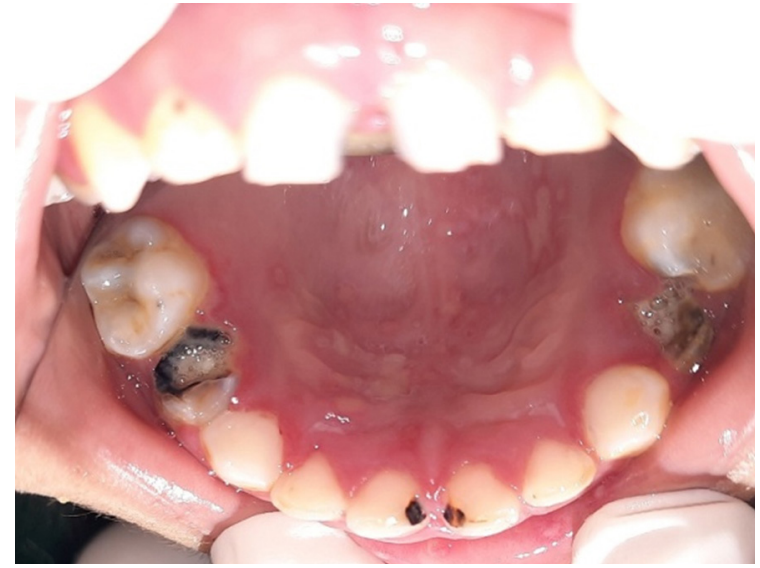

Figure 2. Multiple coalesced ulcers of varying sizes on hard palate.

The patient was instructed to rinse with $15 \mathrm{ml}$ of $0.15 \% \mathrm{w} / \mathrm{v}$ benzydamine hydrochloride solution upto four times per day for 15 days by holding for at least 30 seconds and then expelling from the mouth. The symptoms were relieved within 48 hours (Figure 3,4). The patient was also advised to take soft bland diet and maintain oral hygiene. Though there was complete resolution of symptoms after 15 days, even without the intake of any medications, the whitish coating on the tongue was persistent till one-month followup (Figure 5) and patches of depapillation were still seen in two and three months follow-up (Figure 6,7).

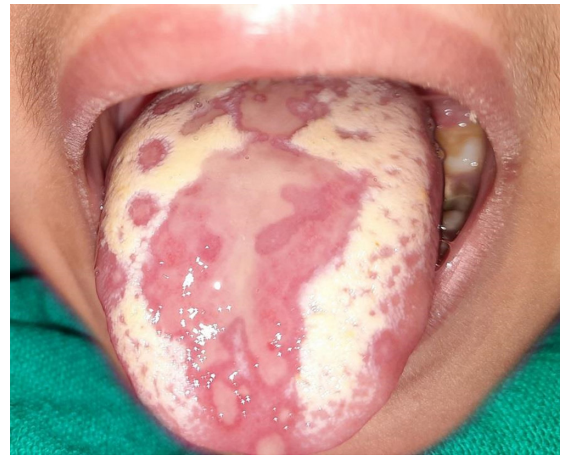

Figure 3. Tongue 48 hours after starting the use of $0.15 \% \mathrm{w} / \mathrm{v}$

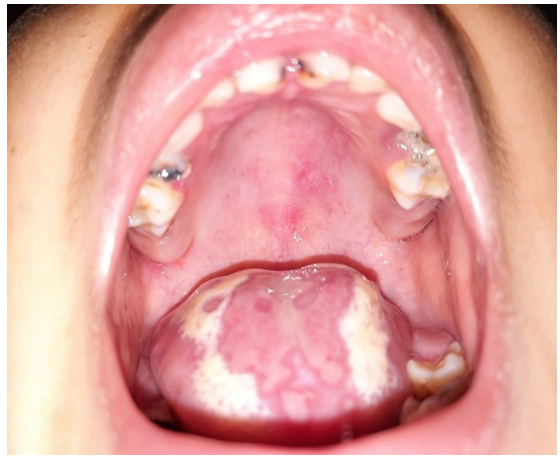

Figure 4. Hard palate 48 hours after starting the use of $0.15 \% \mathrm{w} / \mathrm{v}$

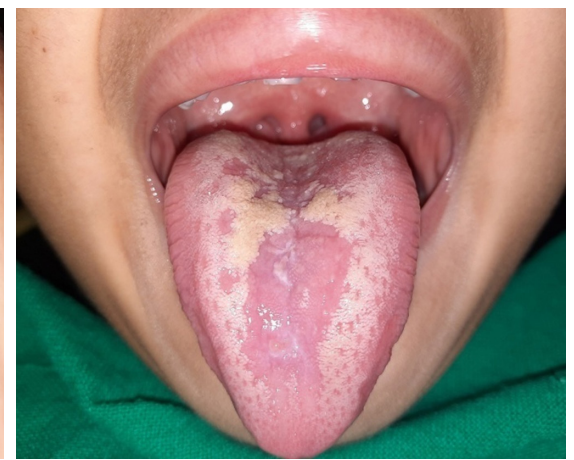

Figure 5. One month follow-up. benzydamine hydrochloride solution. benzydamine hydrochloride solution.

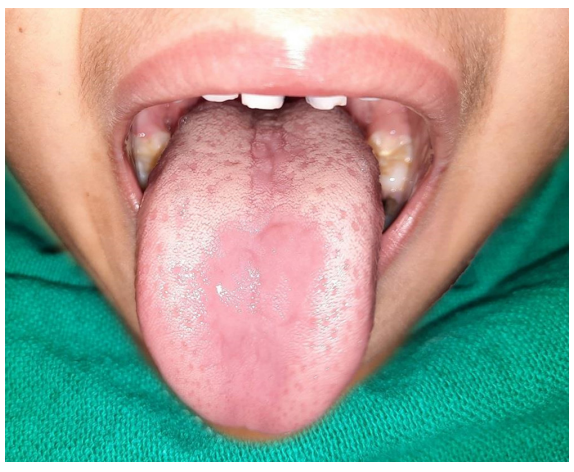

Figure 6. Two months follow-up.

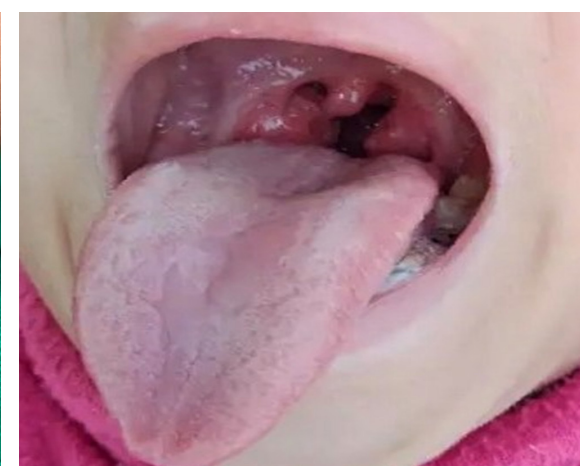

Figure 7. Three months follow-up. 


\section{DISCUSSION}

Pediatric patients having tongue ulceration with white coating can present a diagnostic challenge and dilemma can arise especially when a child,such as in this case,does not exhibit classical presentation of a specific condition, disease, or a syndrome. ${ }^{4}$ Oral candidiasis and the oral manifestation of HIV were ruled out based on clinical examination and laboratory investigations.

Benign migratory glossitis (BMG) also known as geographic tongue, is a benign, inflammatory disorder seen mostly on the dorsal surface of the tongue. It is characterized by presence of constantly changing patterns of multifocal, irregular erythematous patches surrounded by a slightly elevated,keratotic band or line. The lesions can vary from a few millimeters to several centimeters in size. ${ }^{5}$ They are usually asymptomatic but few cases with symptoms like pain, burning sensation, discomfort, sensitivity to hot, spicy, and sour food, pain in ears, or ipsilateral submandibular lymphadenopathy have been reported. ${ }^{6}$ In the present case as well, the patient had a burning sensation, pain in swallowing, and submandibular lymphadenopathy. The white areas on the dorsum of the tongue did not wipe off and exfoliative cytology did not demonstrate any fungal hyphae. However, the lesions did not appear to migrate with time causing a dilemma in diagnosing the case as symptomatic benign migratory glossitis.

Primary herpetic gingivostomatitis (PHG) is a common pediatric infection usually seen in children before the age of 6 years though it can occur in adolescents and adults as well. Though the infection may be asymptomatic in some cases,most of the patients present with symptoms including fever, chills, headache, irritability, pain associated with intake of food and liquids, and malaise. ${ }^{3}$ The most common oral manifestation is the presence of yellow or white liquidfilled vesicles which rupture to form painful ulcers,usually 1 to $3 \mathrm{~mm}$ diameter. The ulcers can be observed on any part of the oral mucous membrane including gingiva, buccal mucosa, tongue, lips, hard and soft palate and the tonsillar areas. Occasionally, large ulcerated lesions may be noted on the palate, gingival tissues, or in the region of the mucobuccal fold. ${ }^{3}$ Despite being a self-limiting disease with spontaneous healing within 10-14 days,symptomatic treatment is recommended to reduce fever and control pain. A soft bland diet with adequate fluid intake is important. Acyclovir is helpful especially in immune compromised patients or in otherwise healthy patient if seen early in the course of the disease. ${ }^{2}$ However, it's found that it does not significantly reduce the duration of fever,length of hospital stay, ${ }^{7}$ and frequency of subsequent recurrences. ${ }^{2}$ In this case,the lesions lasted for a relatively longer period of time which contradicts the usual healing time of PHG. Also, the white coating was seen on the tongue which is not a common feature of PHG.

The PFAPA (Periodic Fever, Aphthous Stomatitis, Pharyngitis, cervical Adenitis) syndrome is an early childhood disease (especially below 5 years of age) with slight male predominance, although the reports of adult-onset disease have markedly increased recently. It is characterized by episodes of fever which usually last for 3-6 days and recur every 3-8 weeks, associated with at least one of the three main symptoms: aphthous stomatitis, cervical adenitis, and pharyngitis. ${ }^{9}$ Patients are usually asymptomatic between the attacks and growth and development is normal. The aphthous lesions of PFAPA syndrome are generally $<1 \mathrm{~cm}$, painful, shallow ulcers with well-defined erythematous borders located on nonmasticatory surfaces of the oral cavity. ${ }^{8}$ Lymphopenia, neutrophilia, monocytosis, raised ESR and a marked increase in CRP levels are recognized during an acute episode of PFAPA in children. ${ }^{8}$ The disease is self-limited requiring only symptomatic treatment for acute attacks. In most cases, antibiotics appear to be ineffective. A single dose of oral corticosteroids ( $2 \mathrm{mg} \mathrm{kg}$ ) has been found to cease the acute attack of PFAPA but does not affect the recurrence of the disease, however the severe side effects of steroids in young patients strongly limit its application. ${ }^{8}$ Since an accurate history of periodic fever associated with at least one of the three main symptoms could not be elicited,thus a definite diagnosis of PFAPA syndrome could not be reached.

In this case, the patient did not demonstrate the characteristic clinical or laboratory findings to support the definite diagnosis of any known condition. As the lesions started healing shortly after the symptomatic treatment, biopsy of the lesion was not performed. This is the first known case till date,to the best of the authors' knowledge, in which a child presented with tongue ulceration with extensive white coating along with fever, tonsillitis, and submandibular lymphadenopathy. Though the symptoms subsided within 48 hours, the lesions lasted for a relatively longer period of time. 
In many countries, parents first turn to pediatric dentists or general dentists for any oral symptoms in their children. Despite having a sound expertise, the broad scope and fast pace of Pediatric Dentistry may limit the dentist's knowledge when encountered with rarer oral mucosal lesions or unusual presentation of common lesions. ${ }^{10}$

\section{SUMMARY}

Tongue lesions can present a diagnostic dilemma as they can be the result of a wide array of disorders ranging from local trauma; infections; immunological and druginduced reactions; malignancy to systemic diseases. Pediatric dentists should be aware of the diversity and management of mucosal anomalies prevalent in children but all oral mucosal lesions do not clearly fall within the scope of Pediatric Dentistry thus necessitating the role of a multidisciplinary approach. Common oral conditions are promptly diagnosed and managed, while those afflicted with rarer and potentially more severe disorders are referred to appropriate specialists for management. This case can be used as a reference for similar lesions which can be encountered in the future and hence help in overall quality management of patient and avoid the overuse of medications

Conflict of Interest: None

\section{REFERENGES}

1. Patil S,Kaswan S,Rahman F,Doni B. Prevalence of tongue lesions in the Indian population.J Clin Exp Dent. 2013 Jul 1;5(3):e128-32. [PubMed | Full Text | DOI]

2. Scully C,Felix DH. Oral medicine-update for the dental practitioner. Aphthous and other common ulcers. Br Dent J. 2005 Sep 10;199(5):259-64. [PubMed I Full $\underline{\text { Text }}$ | $\underline{\text { DOI] }}$

3. Dean JA,editor. McDonald and Avery's dentistry for the child and adolescent. 10 $0^{\text {th }}$ ed. Missouri: Mosby; 2015. [ [ Full Text | Link]

4. Beiraghi S,Myers SL,Regelmann WE,Baker S. Oral manifestations of a possible new periodic fever syndrome. Pediatr Dent. 2007 Jul-Aug;29(4):323-6. [PubMed | Full Text]

5. Sigal MJ,Mock D. Symptomatic benign migratory glossitis: report of two cases and literature review. Pediatr Dent. 1992 Nov-Dec;14(6):392-6. [PulbMed I Full Text]

6. Khadka A,Aryal E. Benign migratory glossitis in toddlers: Report of two cases and review. Nepal Journal of Dermatology Venereology and Leprology. 2020;18(1):8790. [ Full Text| DOI]

7. Huang CW,Hsieh CH,Lin MR,Huang YC. Clinical features of gingivostomatitis due to primary infection of herpes simplex virus in children. BMC Infect Dis. 2020 Oct 20;20(1):782. [ubMed | Full Text | DOI]

8. Batu ED. Periodic fever,aphthous stomatitis,pharyngitis,and cervical adenitis (PFAPA) syndrome: main features and an algorithm for clinical practice. Rheumatol Int. 2019 Jun;39(6):957-970. [․

9. Vanoni F,Theodoropoulou K,Hofer M. PFAPA syndrome: a review on treatment and outcome. Pediatr Rheumatol Online J. 2016 Jun 27;14(1):38. [PulbMed I Full Text | DOI]

10. Hong CHL,Dean DR,Hull K,Hu SJ,Sim YF,Nadeau C,Gonçalves S,Lodi G,Hodgson TA. World Workshop on Oral Medicine VII: Relative frequency of oral mucosal lesions in children,a scoping review. Oral Dis. 2019 Jun;25 Suppl 1:193-203. [PubMed I DOI] 